\title{
Review
}

Luca Carletti*, Marco Gandolfi, Davide Rocco, Andrea Tognazzi, Domenico de Ceglia, Maria Antonietta Vincenti and Costantino De Angelis*

\section{Reconfigurable nonlinear response of dielectric and semiconductor metasurfaces}

https://doi.org/10.1515/nanoph-2021-0367

Received July 12, 2021; accepted September 21, 2021;

published online October 20, 2021

\begin{abstract}
Optically resonant dielectric and semiconductor metasurfaces are an emerging and promising area of nanophotonics and light-matter interaction at the nanoscale. Recently, active tuning of the linear response and nonlinear effects of these components has received an increasing amount of interest. However, so far these research directions have remained separated with only few sporadic works that study their combination beginning to appear in the literature. The evolution of nonlinear metasurfaces based on dielectric and semiconductor materials toward reconfigurable and dynamic components could potentially answer the demand of integrated on-chip components that realize essential functionalities such as frequency conversion, active switching, optical isolation, and all-optical routing. This review provides an overview of recent investigations in this field, reviews the main physical phenomena enabling the dynamic control of the nonlinear response and compares the temporal dynamics of the diverse approaches that have been explored so far. Finally, future directions of dynamic nonlinear metasurfaces are outlined.
\end{abstract}

Keywords: dielectric metasurfaces; nanophotonics; nonlinear optics; second-harmonic generation; thirdharmonic generation.

\footnotetext{
*Corresponding authors: Luca Carletti and Costantino De Angelis, Department of Information Engineering \& CNR-INO, University of Brescia, Brescia 25123, Italy, E-mail: luca.carletti@unibs.it

(L. Carletti), costantino.deangelis@unibs.it (C. De Angelis). https:// orcid.org/0000-0001-6268-9817 (L. Carletti)

Marco Gandolfi, Davide Rocco, Andrea Tognazzi, Domenico de Ceglia and Maria Antonietta Vincenti, Department of Information Engineering \& CNR-INO, University of Brescia, Brescia 25123, Italy. https:// orcid.org/0000-0001-7700-9255 (M. Gandolfi). https://orcid.org/ 0000-0002-5678-0531 (D. Rocco)
}

\section{Introduction}

Sub-wavelength nanoparticles made of high refractive index materials support a rich variety of optical resonances enabling strong electric field confinement and local intensity enhancement [1]. Homogeneous and inhomogeneous periodic distributions of such nanoparticles have been used to realize thin devices - also known as metasurfaces - with unprecedented light manipulation capabilities [2,3]. The optical resonances of high refractive index nanoparticles arise due to the geometry of the nanoparticle itself and are commonly referred to as Mie-type resonances [1]. Magnetic and electric dipole modes and composite higher-order multipoles demonstrated a new route to enhance the nonlinear optical response at the nanoscale [4-8]. Recently, the intriguing bound states in the continuum physics have been applied in this context, demonstrating the possibility to enhance further light-matter interaction at the nanoscale [9-11]. In comparison with metallic nanoparticles that supports collective oscillations between the electric field and electrons at the metal surface [12, 13], dielectric and semiconductor nanoparticles can exhibit optically resonant behaviour without featuring optical absorption in the material. The transparency of dielectric and semiconductor nanoparticles for incident light allows the incident electric field to permeate the nanostructure and increase laser damage threshold. These aspects have been crucial in the field of nonlinear nanophotonics with high refractive index nanoparticles since, from one side, it grants access to the nonlinear response of the whole nanoparticle volume, and, from the other side, it permits using high peak optical intensity to enhance the nonlinear response of the material. Such combination led to the demonstration of second-harmonic generation (using materials with a strong $\chi^{(2)}$ non-linearity such as gallium arsenide, gallium phosphide, and lithium niobate) [11, 14-19] and third-harmonic generation (using materials with a strong $\chi^{(3)}$ non-linearity such as silicon and germanium) [10, 20-23] with recordhigh conversion efficiency compared to other systems of nanometric dimensions. 
Despite the rapid progress of high refractive index nonlinear nanophotonics, almost all demonstrated nanostructures and metasurfaces exhibit a static nonlinear response, i.e. the characteristics such as amplitude, phase, and polarization of the nonlinear optical response are fixed by the fabrication and cannot be changed. However, the strong light-matter interaction in such systems paves the way for a dynamic control of the optical nonlinear response via modification of optical or geometrical properties. This research area is currently emerging in the field of metamaterials and metasurfaces [2,24-28] driven by contemporary and future applications such as optical/quantum communication and computing, virtual or augmented reality, light detection and ranging (LIDAR), and holography. However, so far it remains mainly restricted to consider the effects of the modulation solely on the incident beam: i.e. the nanoparticle or the metasurface acts as a passive system and the output frequency of light equals the input frequency. Nevertheless, metasurfaces based on high refractive index materials also exhibit strong nonlinear optical response that enable generation of a signal at a frequency that is different from the one of the incident beam: i.e. the metasurface acts as an active system that provides signal at a new frequency compared to the input one. The evolution of nonlinear nanoresonators and metasurfaces based on dielectric and semiconductor materials towards reconfigurable and dynamic components could potentially answer the demand of integrated on-chip components that realize essential functionalities such as frequency conversion, active switching, and all-optical routing. The current state-of-the-art is mainly composed of fundamental studies aimed to evaluate the physical mechanisms at play and to assess the achievable time-scale dynamics that are enabled by different approaches. In this review we report the latest advancements in this field and compare the main features of the various physical mechanisms that have been exploited so far.

\section{Tuning mechanisms}

There exist different physical mechanisms that can be exploited to achieve a temporal variation of the optical response of a nanoparticle or a metasurface. Different applications require device reconfigurability within application-specific requirement-based time-scales: 3Dscanning and LIDAR application may be realized to operate up to $\mathrm{MHz}$ rates, while optical communication applications may require $\mathrm{GHz}$ modulation capabilities. Depending on the specific phenomena at play, various modulation rates spanning from the $\mathrm{kHz}$ range up to the $\mathrm{GHz}$ range could be achieved as it is conceptually shown in Figure 1.

Devices with sub-kHz or few $\mathrm{kHz}$ modulation rates can be realized using liquid crystals (LCs). The nonlinear response modulation follows from a change of the optical resonances due to modification of the environmental refractive index. As the birefringence in LCs cells is obtained by rotation of large particles, the modulation rate is inherently low. Up to $\mathrm{MHz}$ modulation rates can be obtained exploiting the thermo-optical effect. When semiconductor materials experience a temperature increase, their refractive index changes in direct proportion to the temperature increased. Being the thermo-optic coefficient small, the temperature variation needed for a sizeable effect is typically few tens of degrees. Higher modulation rates can be potentially obtained using PCM. These feature a dramatic change of electric permittivity at the onset of a specific temperature. If properly operated close to the transition temperature, the necessary temperature variation is reduced and they can potentially lead to modulation rates in the range of few MHz. Ultrafast modulation rates can lastly be achieved by exploiting nonlinear and freeelectrons effects. Here, there are different approaches that can be followed. Nonlinear phenomena such as nonlinear absorption and refraction (either due to third-order nonlinearity or free-carriers) or electric field-induced second-harmonic generation (EFISH) in centrosymmetric materials have been investigated. In the following,

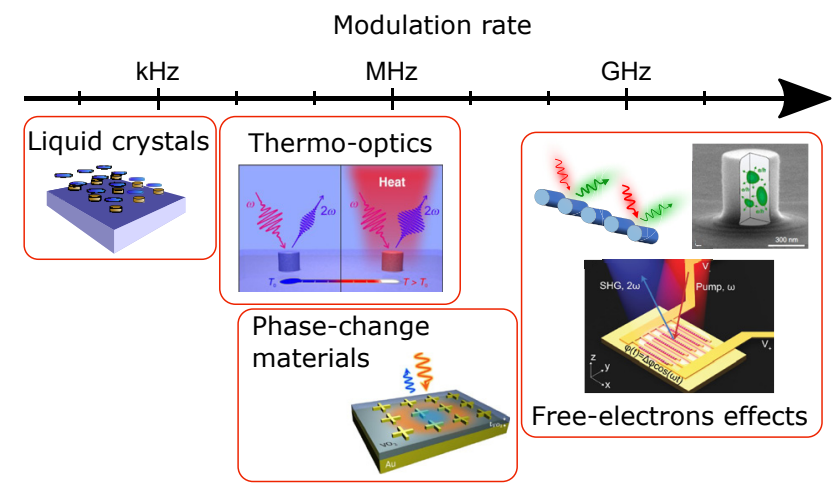

Figure 1: Conceptual representation of different modulation mechanisms as a function of the modulation rate that they allow. Liquid-crystal based devices allow modulation in the $\mathrm{kHz}$ region [29]. Thermo-optic phenomena are limited by thermal diffusion processes and are limited to $\mathrm{MHz}$ modulation rates [30]. Phase-change materials (PCM) exploit almost instantaneous variation of the optical properties of the constituent materials and $\mathrm{MHz}$ modulation rates can be foreseen. Optical tuning via nonlinear effects [31, 32] and electron-induced phenomena [31,33] that are instantaneous can be exploited for realizing devices operating at $\mathrm{GHz}$ speeds. 
these different phenomena and the results that have been achieved are presented.

\subsection{Liquid crystals}

The Mie modes excited in high refractive index nanoparticles such as magnetic dipoles or electric dipoles typically exhibit leaky tails that extend outside the nanoparticle itself. Nonetheless, a moderate field enhancement is obtained in proximity of the nanoresonators. This entails the possibility to change the optical response of the system by modification of the environmental refractive index. In this context, the use of LCs is an attractive approach because it relies on the well-established expertise from the LC display industry [34-40]. LCs have an optical birefringence that can be tuned by applying an external electric field or by increasing the working temperature [41]. Recently, silicon-based metasurfaces infiltrated with LCs have demonstrated spectral tuning of Mie resonances [42] and dynamic beam-steering [43]. In the visible range, a hybrid metasurface-spatial light modulator (SLM) system has been realized using titanium dioxide $\left(\mathrm{TiO}_{2}\right)$ metasurfaces [44]. This phase-only nanoantenna-based transmissive SLM device reached an experimental efficiency of $36 \%$ with a pixel size of only $\approx 1 \mathrm{~mm}$ and a field of view (FOV) of $22^{\circ}$. In [29], Rocco et al. show the design of a dielectric metasurface of AlGaAs nanodisks, embedded in an LC material, and layered over an AlOx substrate to achieve second-harmonic (SH) intensity modulation as well as a reconfigurable $\mathrm{SH}$ directivity, see Figure 2.

Intuitively, the LC anisotropy is associated with a consistent modification in the linear transmission of the (a)

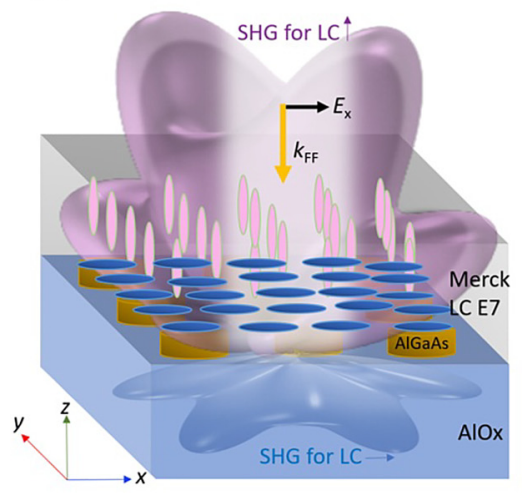

(c)

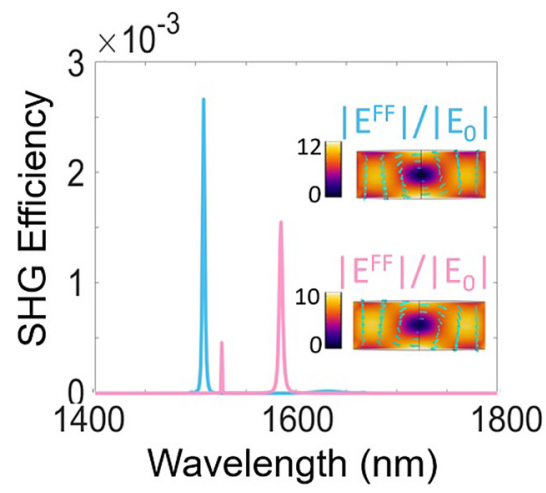

(b)

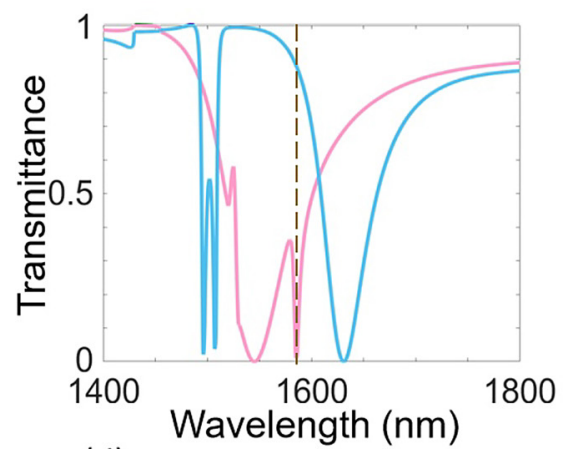

(d)

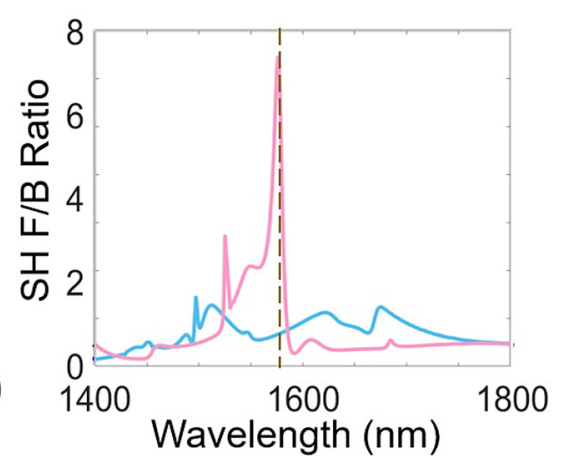

Figure 2: SHG in AlGaAs metasurface infiltrated with liquid-crystals.

(a) Pictorial representation proposed metasurface. The unitary cell is made of an AlGaAs nanodisk over an AlOx substrate embedded in LCs. (b) Numerical calculations of the spectral resonances for the two LC alignments: the light blue curve is associated to the LC directors parallel to the $x$ axis (planar orientation) whereas the pink curve is related to the LC directors parallel to the $z$ axis (homeotropic orientation). The brown dotted line indicates the MD resonant wavelength in the case of LC homeotropic orientations. (c) SHG efficiency as a function of the incident wavelength for the two LC orientations. The upper inset corresponds to the plot of the electric field enhancement at the MD resonance for the homeotropic LC alignment; the lower one represents the enhancement at the MD resonance at $1508 \mathrm{~nm}$ for the $\mathrm{LC}$ planar orientation. (d) Theoretical calculations of the SH F/B ratio as a function of the fundamental wavelength for the two LC orientations: the light blue line refers to the LC directors parallel to the $x$ axis, instead the pink curve stems for the LC directors parallel to the $z$ axis. Reproduced from [29]. 
metasurface with respect to the homogeneous isotropic surrounding material. Solely, the AlGaAs nanodisks are responsible for the SHG since the LC and the substrate have both a negligible second-order nonlinear susceptibility. Thus, the SH signal coming from the entire metasurface can be engineered by a proper excitation of the AlGaAs nanodisk modes.

For a geometrical-optimized structure operating in the third communication window (wavelength range 1400-1800 $\mathrm{nm}$ ), the magnetic dipole (MD) resonance of the metasurface occurs at a wavelength of $1508 \mathrm{~nm}$ when the LCs are in a planar alignment and at a wavelength of $1580 \mathrm{~nm}$ for the LCs homeotropic orientation as shown in Figure 2(c)). Hence, since enhancement of SHG are observed when the metasurface is excited at the MD resonance, the spectral position of the peak SHG conversion efficiency is controlled by the orientation of the LCs. Alternatively, for a pump operating at a fixed polarization and wavelength, by tuning the LCs orientation, an SH intensity modulator is addressed.

Interestingly, the $\mathrm{SH}$ front-to-back $(\mathrm{F} / \mathrm{B})$ ratio for $\mathrm{LC}$ homeotropic alignment (pink curve of Figure 2(d)) is as high as 6.5 around the SHG efficiency peak due to the MD resonance. For the same wavelength, the F/B ratio for the other LC orientation is equal to 0.65 . Thus, around a pump wavelength of $1580 \mathrm{~nm}$, a $10 \mathrm{~dB}$ enhancement in the $\mathrm{SH}$ $\mathrm{F} / \mathrm{B}$ ratio is obtained for the two considered LC orientations. This demonstrates that LC anisotropy offers the capability to turn on and off the SH signal and simultaneously the possibility to tune the $\mathrm{SH}$ F/B ratio of the metastructure.

The main limitation in the performance of such LCbased devices is related to the response time of the liquid crystals itself. Indeed, the LC cell is an intrinsically slow device with rather long switching times $(\approx 50 \mu$ s) [45]. When an external electric field is set, the molecules are forced to align according to the electric field, while when the electric field is reset, the molecules return to their natural position. Another aspect to take into account is that the alignment of the molecules induced by the electric field is faster than their subsequent internal rearrangement. These relatively slow responses are mainly dominated by the viscosity of the liquid crystal and depend on the applied electric field, the temperature and the type of molecule (with lesser or greater polarity).

\subsection{Thermal effects}

The nonzero imaginary part of the refractive index implies light absorption within the materials. Hence, in this regime, the light absorbed energy is converted into heat and a thermal dynamics is triggered [46, 47], resulting in a temperature increase within the system [48]. In particular, by exploiting the highly confined electric field obtained with Mie resonances, efficient light-to-heat conversion has been demonstrated in metallic and dielectric nano-objects [24, 49-58]. The interplay between the optical and the thermal response in the nanostructures and metasurfaces may be used to actively tune the system's physical properties [59]. In particular, the system's temperature increase, obtained upon light illumination or simply by placing the structure in contact with a hot thermal bath, leads to a modification of the materials' refractive index, thus altering the optical behavior of the investigated structure. The latter phenomenon is known as thermo-optical effect and the refractive index variations $\Delta n$ are directly proportional to the temperature increase $\Delta T$ via the thermo-optic coefficient $\mathrm{d} n / \mathrm{d} T$ :

$$
\Delta n(\lambda)=\frac{\mathrm{d} n}{\mathrm{~d} T}(\lambda) \Delta T,
$$

where $\Delta T=T-T_{0}$ is the temperature increase with respect to the environment one $T_{0}$, and $\lambda$ is the light wavelength.

The tuning of the SHG in isolated AlGaAs nanodisks via the thermo-optical effect has been recently reported [30]. The thermo-optic coefficient for AlGaAs is relatively low $\left(<10^{-4} \mathrm{~K}^{-1}\right)$ in the near-infrared range, whereas it increases exponentially in proximity of the bandgap wavelength $(\sim 750 \mathrm{~nm})$ [60]. Therefore, exploitation of thermooptic effect can be efficient for modulation of the nonlinear response when either the pump or the generated signal is close to this wavelength. Indeed, Celebrano et al. designed an SHG experiment in AlGaAs nanodisks with a pump at a wavelength of $1550 \mathrm{~nm}$. By increasing the nanodisk temperature by a few $\mathrm{K}$, a negligible refractive index variation is obtained at the pump wavelength, whereas the refractive index at the second-harmonic $(\sim 775 \mathrm{~nm})$ changes significantly. This yields a strong dependence of the SHG efficiency on the nanodisk temperature. Indeed, as the refractive index increases, the optical resonances of the nanodisk typically red-shift, thereby spectrally shifting the peak SH conversion efficiency. The SHG modulation from a fixed pump wavelength of $1550 \mathrm{~nm}$ can thus be controlled by the radius of the nanodisks as shown in Figure 3(a).

In [30], two strategies to demonstrate the thermo-optic effect are proposed: (i) heating the sample with a Peltier cell or (ii) illuminating the sample with a control continuouswave laser beam at a wavelength of $405 \mathrm{~nm}$ which is in the AlGaAs absorption region. Provided that the power of the control beam is tuned to yield the same nanodisk average temperature increase as the one obtained heating with the Peltier cell, the obtained modulation of the SHG 

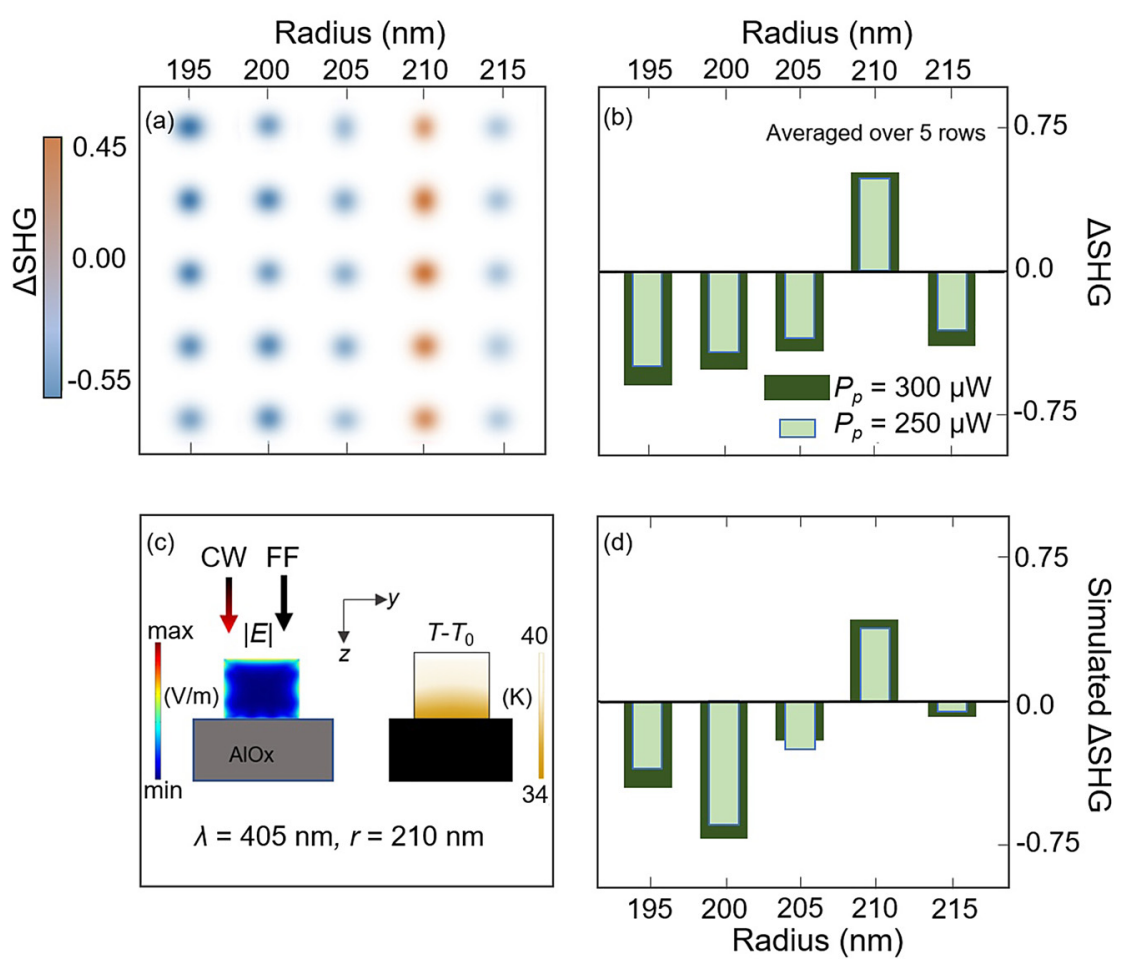

Figure 3: Thermal tuning of SHG in AlGaAs nanodisks.

(a) Measured differential SH signal $\left(\triangle \mathrm{SHG}\left(P_{p}\right)\right)$ collected scanning five replicas (on the same column) of five different nanodisk (5 lines) with a control signal power, $P_{p}$, of $300 \mu \mathrm{W}$. (b) Experimental $\Delta \mathrm{SHG}\left(P_{p}\right)$ for five different nanodisk radii for two control powers $\left(P_{p}=250 \mu \mathrm{W}\right.$ and $P_{p}=300 \mu \mathrm{W}$ ). (c) Schematic of the electric field (left) and induced temperature field in the nanodisk (right) when

$P_{p}=300 \mu \mathrm{W}$. (d) Theoretical calculations to be compared with the experimental measurements of panel (b). Reproduced from [30].

is equivalent for both configurations (see Figure 3(b)-(d)). Small differences in the SHG modulation of the two configurations are due to the different spatial distribution of the temperature inside the nanodisk, Figure 3(c). However, as long as the average nanodisk temperature is the same, the measured SHG modulation is quite similar.

While the rising time of the thermo-optical effect is typically within $1-10$ ps [61], the relaxation process is governed by phonon-phonon scattering and, in nanostructures, it can be as low as $100 \mathrm{~ns}[24,30]$. These response times may enable the realization of reconfigurable thermo-optic nonlinear metasurfaces operating up to a few $\mathrm{MHz}$ modulation rates.

\subsection{Phase-change materials}

The tuning mechanism of PCM is based on the variation of the optical properties, i.e. real and imaginary part of the refractive index, across the phase transition. Depending on the material, the involved phase transition can drive the material from a dielectric to a metallic phase, as for $\mathrm{VO}_{2}$, or from a crystalline to an amorphous arrangement of the crystal structure, like in GeSbTe (GST) alloys.
Although PCMs have been extensively investigated to tune the linear electromagnetic response of photonic structures, such as thin films, gratings, ring cavities, nanoantennas and metasurfaces [27,62], their use in the control of the nonlinear response is still in its infancy and it has very recently emerged only in the framework of plasmonic devices. Among the most investigated materials for nonlinear optics, chalcogenides have a history of decades, and have provided a number of applications in electronics and photonics [63]. In particular, PCM chalcogenides, based on GST alloys, are known to possess both very large modulation of optical properties and very large nonlinear susceptibilities. In [66], nonlinear absorption and selffocusing refraction coefficients up to $-6.63 \times 10^{-2} \mathrm{~m} / \mathrm{W}$ and $2.606 \times 10^{-9} \mathrm{~m}^{2} / \mathrm{W}$, respectively, were demonstrated in thin $\mathrm{PCM} \mathrm{Sb}_{2} \mathrm{Te}_{3}$ films with z-scan (at $632.8 \mathrm{~nm}$ ) and temperature-dependent ellipsometry. A theoretical investigation of the experimental results elucidated that the nonlinear absorption is due to the band filling effect, while the nonlinear self-focusing effect originates from the bandgap shrinking. A tunable nonlinear response has been experimentally observed also in $\mathrm{VO}_{2}$ nanoparticles and thin films. Even though the transition from monoclinic 
to rutile does not change significantly the $\mathrm{VO}_{2} \chi^{(3)}$ tensor symmetry, the change in the linear polarizability of $\mathrm{VO}_{2}$ molecules induces a change of the nonlinear coefficients. Indeed, the Miller's rule, which establishes how the nonlinear susceptibility of order $n+1$ scales with the linear susceptibility [67]:

$$
\begin{aligned}
& \chi^{(n+1)}\left(\omega_{n+1}, \omega_{n}, \ldots, \omega_{1}\right) \\
& =A\left[\chi^{(1)}\left(\omega_{n+1}\right) \chi^{(1)}\left(\omega_{n}\right) \ldots \chi^{(1)}\left(\omega_{1}\right)\right],
\end{aligned}
$$

clearly suggests that the strong change of linear optical properties in PCMs ( $\mathrm{VO}_{2}$ and GST alike) goes hand in hand with a strong modulation of the nonlinear susceptibility. Petrov, Yakovlev, and Squier in [68] demonstrated a 1.5orders of magnitude increase of THG from $\mathrm{VO}_{2}$ thin films induced both thermally and optically with ultrafast laser pulses, corroborating, experimentally, the idea that the phase transition in PCM alters both linear and nonlinear response. Z-scan and ultrafast pump-probe experiments in $\mathrm{VO}_{2}$ nanoparticles and thin films revealed that in the metallic state, $\mathrm{VO}_{2}$ exhibits a positive nonlinear index of refraction with nanoparticles showing larger nonlinearity. Saturable absorption effects are measured in the metallic state of nanoparticles while positive nonlinear absorption coefficients are retrieved for thin films in the metallic state. Interestingly, saturable absorption switches to nonlinear absorption (two-photon absorption) in the semiconducting phase of nanoparticles, while no change of sign of the nonlinearity is experienced by thin films. On the other hand, a change of sign is measured in the z-scan measurement of the nonlinear refractive index of thin films, which switches from positive in the metallic state (self-focusing) to negative in the semiconducting state (self-defocusing). The strong variation of nonlinear response at the phase transition has also been used to unveil the dynamics of the phase transition in PCMs. In [69], Lysenko et al. performed degenerate four wave mixing, pump-probe experiments to elucidate the structural dynamics of both thermally- and light-induced phase transition in $\mathrm{VO}_{2}$ films, finding coexistence of both phases and fluctuations of the dielectric constant. A significant decrease of the $\mathrm{VO}_{2}$ third-order nonlinear susceptibility was measured. Similar behavior was found in nanostructured $\mathrm{VO}_{2}$ thin films [70].

Tognazzi et al. in [71] proved that a small gap filled with PCM is a very efficient way to tune the behavior of a plasmonic nanoantenna in the linear regime. In [64], Guo, Zhou, and Guo extended this concept to the nonlinear regime and they theoretically demonstrated tuning of second harmonic generation in the infrared by using a periodic metasurface of gold split ring resonators with gaps filled with GST (see Figure 4(a)). The crystalline fraction of GST was thermally controlled and it was proved to significantly affect the $\mathrm{SH}$ wavelength, with larger $\mathrm{SH}$ wavelengths for smaller gaps and larger crystalline fractions. In this work the contribution due to the second order nonlinear susceptibility tensor components of GST are neglected and the tuning mechanism is due to the modulation of the electric field distribution at the fundamental frequency, which significantly affect the surface plasmon resonance and thus the SHG. In [65] GST sandwiched between a gold substrate and a gold nanodisk allowed to increase and tune THG (see Figure 4(b)). The presence of GST increased the TH by 5 orders of magnitude due to the large bulk $\chi^{(3)}$ of GST and the large field enhancement provided by the structure on resonance. The THG tuning was ascribed to two effects: (i) the strong change of field enhancement in GST associated with the large variation of the (linear) refractive index and the consequent shift of the plasmonic resonance; (ii) the large modulation that the GST $\chi^{(3)}$ undergoes when it transitions from the amorphous to the crystalline state (the susceptibility indeed varies by a factor larger than 10 upon transition, from $\sim 10^{-18}$ to
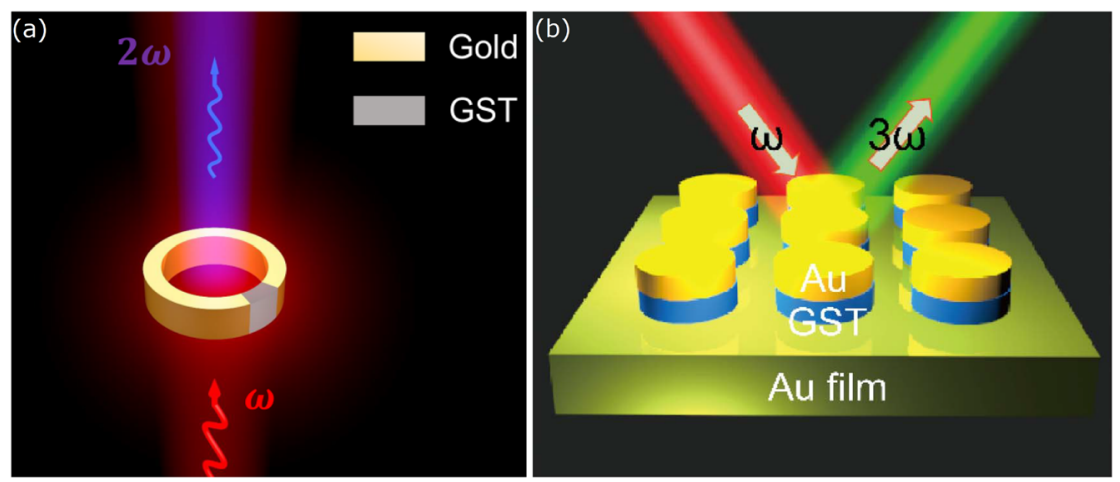

Figure 4: Hybrid gold-GST nanostructures.

(a) Isolated split ring resonator with gap filled with GeSbTe (GST) for tuning SHG by switching from the amorphous to the crystalline phase.

(b) Plasmonic metasurface for THG reconfigurable by GST. Figure adapted from [64, 65]. 
$\sim 10^{-17} \mathrm{~m}^{2} / \mathrm{V}^{2}$ ). In [72], Cao et al. measured THG from an asymmetric, Fabry-Perot thin-film cavity made of a GST film ( $50 \mathrm{~nm}$ thick) separated by a gold substrate by a dielectric spacer (see Figure 5). The results indicated that the field-enhancement at the cavity resonance provided $\sim 400$ fold enhancement of THG with GST in the amorphous state. After thermally-induced crystallization, a drop of THG by three orders of magnitude, was observed, clearly indicating the feasibility of GST-based devices with tunable or reconfigurable nonlinear optical response. Up to $30 \mathrm{~dB}$ modulation of THG has been recently predicted in a Salisbury-type, metasurface with an array of plasmonic nanoantennas separated by a metallic backplane by a $\mathrm{VO}_{2}$ thin film [73].

These results show that the integration of PCMs in resonant photonic nanostructures is very promising for dynamic control of nonlinear effects. Indeed the modulation acts on multiple factors that regulate harmonic generation. In general, the conversion efficiency of $n$-harmonic generation scales as:

$$
\eta_{n} \sim \chi^{(n)}\left(\frac{\left|E_{\text {loc }}\right|}{\left|E_{\text {in }}\right|}\right)^{n}
$$

where $\chi^{(n)}$ is the material nonlinear susceptibility of the order $n$ (e.g. $n$ equals to 2 for SHG and 3 for THG), $\left|E_{\text {loc }}\right| /\left|E_{\text {in }}\right|$ is the ratio between the local electric field amplitude and the input electric field amplitude (i.e. field enhancement). Both the factors in Eq. (3) are significantly modulated in PCM-based photonic nanostructures, with the second factor, i.e. the field enhancement, being especially sensitive to (linear) refractive index variations induced near photonic resonances. In this sense, in [74] the authors employed a $\mathrm{VO}_{2}$ thin film to generate picoseconds electrical pulses to drive a dipole antenna generating broadband $\mathrm{THz}$ radiation. This approach strongly differs from the one employed by Esaulkov et al. in [75], where they tuned $\mathrm{THz}$ radiation directly from a thin $\mathrm{VO}_{2}$ film by exploiting the $\chi^{(2)}$ due to the symmetry breaking at the surface.

The transition time between the two material phases depends on the specific PCM and external stimulus employed. In the electrically heated GST based structures it can be in the nanosecond or even subnanosecond time scale [76-78], while optical excitation via femtosecond pulses in $\mathrm{VO}_{2}$ revealed sub-50 fs transition times [68]. These response times entail the possibility to develop reconfigurable nonlinear metasurfaces operating above $\mathrm{MHz}$ speeds.

\subsection{Ultrafast nonlinear optical effects}

Semiconductors and dielectrics used for SHG and THG demonstrations usually exhibit a strong $\chi^{(3)}$ non-linearity at the pump wavelength. Beside the THG phenomenon, Kerr-effect and two-photon absorption (TPA) are the manifestation of third-order nonlinear effects [67]. The Kerreffect is related to the real-valued part of the third-order nonlinear susceptibility and it manifests as an intensity dependent refractive index variation that is often characterized by the nonlinear refractive index $n_{2}$ :

$$
\Delta n=n+I n_{2}
$$

where $n$ is the refractive index of the unperturbed material and $I$ is the optical intensity. The imaginary part of the $\chi^{(3)}$ nonlinear susceptibility induces a nonlinear absorption phenomenon known as TPA. The effect of TPA on the nonlinear optical response of a nanoresonator is twofold, it lowers the conversion efficiency by increasing the absorption of the pump beam, and it photoinjects free-carriers in the material conduction band that may strongly modify the material optical properties via subsequent free-carrier (FC) effects. The effect of these phenomena in dielectric
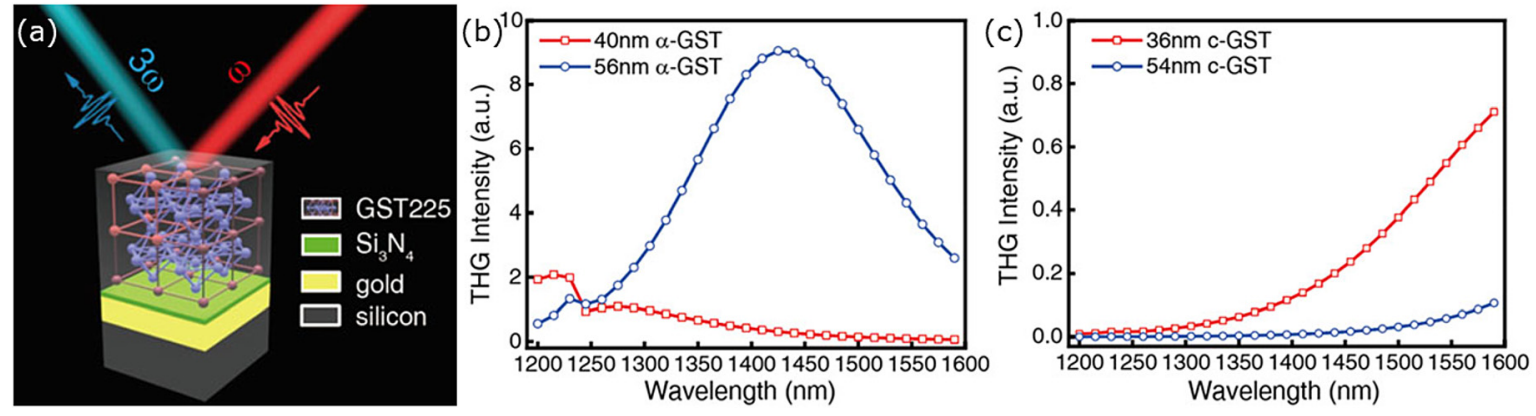

Figure 5: THG modulation in Fabry-Perot thin-film GST cavity.

(a) A GST film is employed to change the THG by switching from the crystalline to the amorphous phase. (b) THG intensity spectrum for two different GST film thicknesses in the amorphous phase. (c) THG intensity spectrum for two different GST film thicknesses in the crystalline phase. For the $56 \mathrm{~nm}$ film, there is one order of magnitude of modulation in THG. Figure adapted from [72]. 
metasurfaces has been studied in both isolated nanoresonators and metasurfaces [31, 79-83]. In [31], the effect of TPA and subsequent FC effects to achieve dynamic control of the SHG in arrays of infinitely long GaAs nanowires is investigated. The geometry is optimized to have an MD resonance at a wavelength of $1800 \mathrm{~nm}$ and a higher-order multipolar resonance at the corresponding SH wavelength (see Figure 6(a)). The pump wavelength is chosen in that range since GaAs exhibits a high TPA coefficient of $6 \times 10^{-11}$ $\mathrm{m} / \mathrm{W}$. The density of FC, $N$, photoinjected in the material due to TPA can be found by solving the rate equation

$$
\frac{\mathrm{d} N}{\mathrm{~d} t}=\frac{\beta I^{2}}{2 \hbar \omega_{\mathrm{FF}}}-\frac{N}{\tau_{\mathrm{r}}}
$$

where $\beta$ is the TPA coefficient, $I$ is the intensity at the pump wavelength, $\tau_{\mathrm{r}}$ is the carrier relaxation time, $\omega_{\mathrm{FF}}$ is the pump angular frequency, and $\hbar$ is the reduced Planck's constant. The presence of FC plasma can lead to an increase of absorption (free-carrier absorption, FCA) and variation of refractive index (free-carrier dispersion, FCD) [67]. Since the FC density, $N$, depends upon the intensity of the pump beam through Eq. (5), the optical response of the nanoresonators and, in turn, the SHG efficiency depend on the pump beam intensity. These effects are numerically investigated using a finite-element-method model that solves self-consistent nonlinear Maxwell equations. This is shown in Figure 6(b). The effect of TPA, neglecting FC effects, is to limit the SHG conversion efficiency due to increased pump absorption. A stronger modulation of the conversion efficiency is observed when FCD and FCA are considered. In particular, FCD strongly reduces the SH conversion efficiency when the pump intensity is beyond $20 \mathrm{GW} / \mathrm{cm}^{2}$ due to a spectral blue-shift of the MD resonance at the pump wavelength.

At frequencies that are close to the electronic energy bandgap of the material, variations of absorption and refraction due to excitation of FC are enhanced by bandgap shrinkage and band-filling effects [84-86]. The bandfilling effect manifests as a decrease in inter-band absorption for photon energies slightly above the bandgap energy. This is a consequence of the excited FC occupying the lowest conduction band states so that the electrons in the valence band require higher energy than the nominal bandgap energy to be excited into an available state in the conduction band. Reversely, bandgap shrinkage manifests as an increase in inter-band absorption for photon energies slightly below the bandgap. This phenomenon arises due to the interactions between electrons in densely populated low-energy states of the conduction band that result in a decrease in their energy. By applying Kramers-Kronig relations, these variations in the material absorption are accompanied by modulations of the refractive index. Figure 6(c) shows the effect of these FC effects on the SHG efficiency. The SHG peak wavelength strongly depends on the pump intensity up to $20 \mathrm{GW} / \mathrm{cm}^{2}$ and it exhibits a complex dynamics that can be explained observing the contribution of each modulation phenomenon. The band-filling and the FCD effect cause a reduction of the resonator refractive index, while the bandgap shrinkage effect induces an increase of the refractive index. This is evident considering a fixed pump wavelength of $1800 \mathrm{~nm}$, Figure 6(d) shows the refractive index modulation phenomena as a function of the pump intensity. For low pump intensities (below $10 \mathrm{GW} / \mathrm{cm}^{2}$ ), (a)

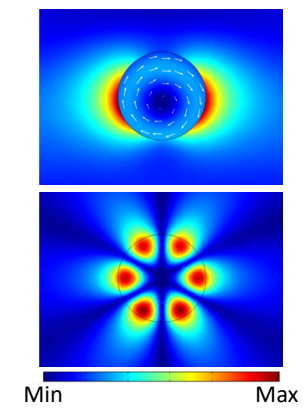

(b)

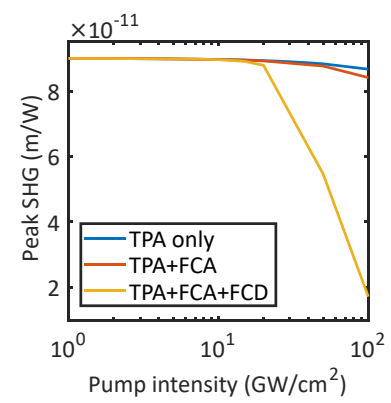

(c)

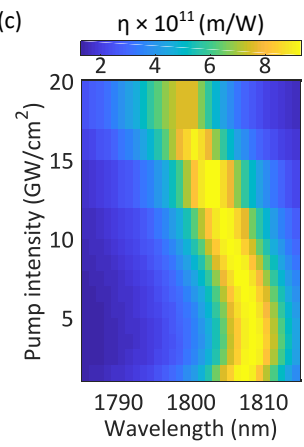

(d)

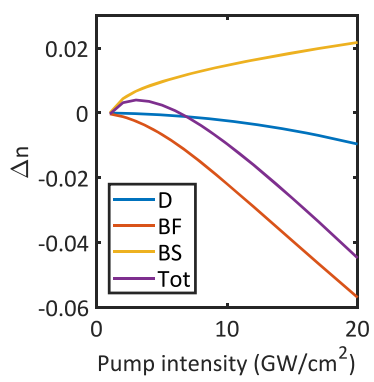

Figure 6: SHG modulation in GaAs nanowires.

(a) Electric field intensity at the pump wavelength (upper panel) and the second-harmonic wavelength (lower panel). (b) Peak SHG conversion efficiency as a function of the pump beam intensity. Note that the conversion efficiency is measured in unit of $\mathrm{m} / \mathrm{W}$ since the analysed structure is two-dimensional and thus all the powers considered have unit of W/m. Only modulation of the material parameters at the pump wavelength is considered. (c) SHG conversion efficiency spectra as a function of pump intensity and pump wavelength, considering modulation of the material parameters at both pump and SH wavelengths. (d) Induced refractive index change at the wavelength of $900 \mathrm{~nm}$ as a function of the pump intensity due to FCD (D), band-filling (BF), bandgap shrinkage (BS) effects. The sum of all the effects (Tot) is also shown. Figure adapted from [31]. 
the overall effect of all these contributions can mostly be attributed to the bandgap shrinkage, whereas at high intensities the band-filling effect becomes dominant. Numerical modeling of these phenomena predicts a modulation of the SHG signal at a fixed wavelength with a contrast up to $60 \%$.

Photoinjection of FCs in the conduction band of a semiconductor can be achieved also exploiting the linear absorption of a control beam at a wavelength in the material absorption spectrum. In [33], the SHG produced in AlGaAs nanodisks from a pump beam at a wavelength of $1550 \mathrm{~nm}$ is modulated using a control beam at a wavelength of $500 \mathrm{~nm}$, see Figure 7(a). The control beam wavelength is in the absorption band of AlGaAs and thus allows photoinjecting $\mathrm{FC}$ in the material and triggers permittivity modulations at the band-edge of the semiconductor. Measurements shown in Figure 7(b) demonstrate a modulation of the SHG generated at a wavelength of $775 \mathrm{~nm}$ up to $6 \%$ with respect to the unperturbed resonator. The dynamics of the SHG modulation is modeled using a perturbative finiteelement-method model that well reproduces the measurements, see Figure 7(c). The alteration of material properties due to photoinjection of FCs is estimated using a twopopulations model: a first population is photoinduced by the control pulse and is spatially distributed as the optical mode that is excited in the nanoresonator. This population
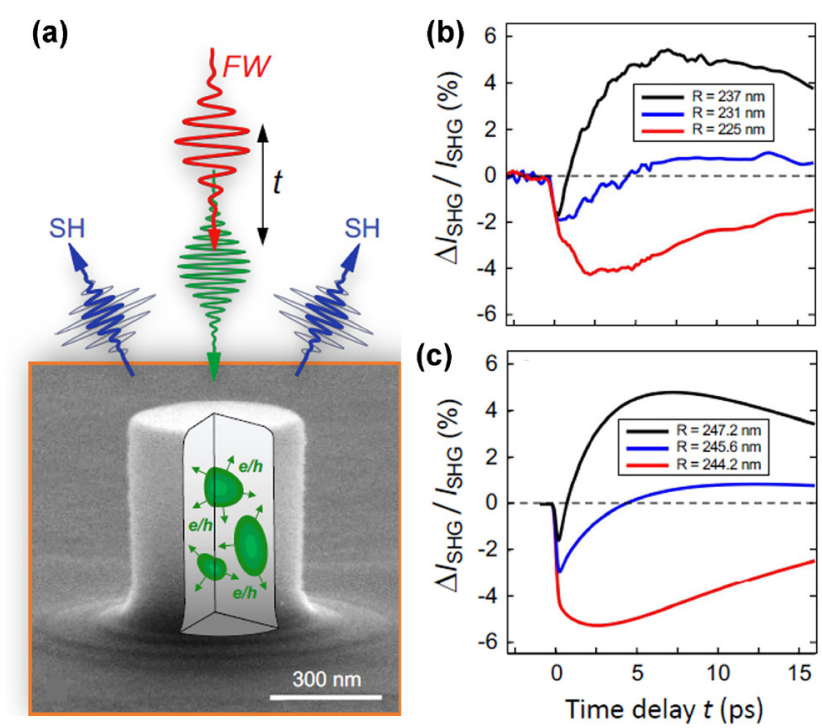

rapidly evolves via ambipolar diffusion process generating a second uniformly distributed population. This second population finally relaxes by non-radiative recombination increasing the temperature of the AlGaAs lattice.

The response times of FC-induced modulations are limited by the relaxation times of FCs. In nanostructures, the presence of a high surface-to-volume ratio reduces FCs recombination times, as such process is enhanced at surfaces, and it is typically in the order of a few picoseconds. This entails the possibility to develop reconfigurable nonlinear metasurfaces operating at and beyond $\mathrm{GHz}$ modulation rates.

Materials with a centrosymmetric lattice such as silicon do not exhibit $\chi^{(2)}$ nonlinearity. Nevertheless, SHG can be achieved via electric field induced second harmonic (EFISH) effect. Here, the application of a bias voltage across the medium allows effective SHG by mixing of the static or low-frequency field with an intense pump beam at optical frequencies. In [32], a silicon-based metasurface resonant at a wavelength of $1550 \mathrm{~nm}$ exploits interference between ultrafast temporal modulation of strong Kerr effect and dc electric field to enhance EFISH intensity, see Figure 7(d). The demonstrated on/off ratio of SHG using a bias voltage of $37 \mathrm{~V}$ is above $40 \mathrm{~dB}$. Ultrafast modulation with an RF signal with a $1 \mathrm{MHz}$ repetition rate is also demonstrated in Figure 7(e).

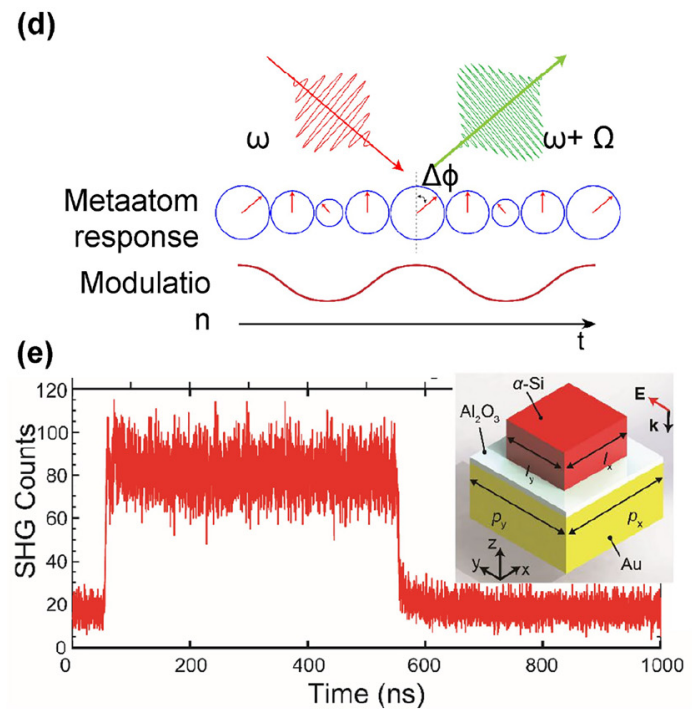

Figure 7: FC-induced SHG modulation and EFISH effect in nanostructures.

(a) Scanning electron microscopy image of an AlGaAs nanodisk and sketch of the all-optical control of SHG via photoinjection of FCs. (b) Measured differential SHG for the three different nanodisks as a function of the time delay between the control and pump pulse. (c) Simulated relative differential SHG for three different sizes (representative of the experiments), (d) principle of dynamically enhanced parametric oscillation in a metasurface. The dynamic interference between dc electric field and fundamental optical field creates a traveling-wave modulation to $\alpha$-Si meta-atoms, leading to SHG with enhanced efficiency. (e) Time-resolved trace of SHG modulated by a square-wave electrical signal (top panel) at $1 \mathrm{MHz}$ repetition rate. A sketch of the metasurface unit cell is shown in inset. Figure adapted from [32, 33]. 


\section{Conclusion and outlook}

In this work, we reviewed the recent development in the field of dielectric and semiconductor nanoresonators and metasurfaces with a tunable nonlinear optical response. This field is rapidly emerging and developing inspired by demonstrations of dynamic control of the linear optical response on such devices. High refractive index resonators offer a unique combination of properties such as highfiled confinement and enhancement, strong nonlinear light-matter interaction, and lossless operation that are particularly favorable for the realization of reconfigurable devices.

The path to the development of nanoresonatos and metasurfaces with tunable nonlinear response such as SHG or THG is still to unravel. Recent theoretical investigations and demonstrations dealt with different physical mechanisms to obtain a nonlinear response that can be reversibly tuned. The use of liquid crystals seems a promising strategy to obtain strong modulations of the SHG signal from a metasurface with modulation rates up to the $\mathrm{kHz}$ range and rely on a technologically mature field. Thermo-optics phenomena have shown the possibility to control the nonlinear response with an all-optical scheme. The slow dynamics of heat diffusion, however, limits the modulation rate in the $\mathrm{MHz}$ range. Although triggered by a temperature increase, PCM offer a method to achieve strong modulation at higher modulation rates provided that the operation temperature of the device is close to the critical temperature of the material. Finally, the nonlinear effects and effects due to free-electron plasma excited in the conduction band of the semiconductors provide an ultrafast approach to modify the optical properties of the resonators. Since the relaxation times of such free-carriers is typically on the order of a few picoseconds, $\mathrm{GHz}$ modulation rates and beyond can be expected. These fast modulation rates have also opened the way for a new class of metasurface based devices known as time-variant metasurfaces that enable frequency conversion and nonreciprocal propagation [7]. In fact, in a material whose optical properties dynamically change over time with a frequency $\Omega$, when illuminated with light at a frequency $\omega$, side bands at frequencies $\omega \pm \Omega$ can be generated. Recently, this phenomenon has been demonstrated in a metasurface at optical frequencies [87]. Similar effects could potentially enable many interesting phenomena in nonlinear semiconductor metasurfaces, such as non-reciprocity and time-bandwidth limitation [88].

Despite a number of already successful results have been achieved, other strategies to achieve tunability of the nonlinear response need to be investigated in the future. For example, if the metasurface is deposited on an appropriate substrate, upon application of a mechanical strain, the distance between nanoresonators can be varied, thereby modulating the optical response [89-91]. Moreover, the elasticity of dielectric materials makes it possible for such nanostructure to exhibit mechanical resonance in the $\mathrm{MHz}-\mathrm{GHz}$ range. The interaction between opto-mechanical modes has proved transmittance and reflectance modulation of metasurfaces from $\mathrm{MHz}$ up to few $\mathrm{GHz}$ modulation rate [92, 93]. Another interesting perspective is the use of two-dimensional materials such as graphene $[47,94,95]$. Such atomically-thin layers could be integrated in metasurfaces where the optical resonances of the metasurfaces could be used to enhance the electric field in the 2D-material and increase light-matter interaction. Finally other electro-optical phenomena will constitute an interesting platform to explore. In particular, materials with strong $\chi^{(2)}$ effects can exhibit the Pockels effect which involves a modulation of the refractive index upon application of a static electric field in the material. Moreover, integration of high refractive index metasurfaces with thin layers of transparent semiconductor oxides (TCO) that exhibit an epsilon-near-zero (ENZ) point close to the wavelength region of interest can potentially lead to strong and ultrafast modulation rates. In this context, the ENZ point of the TCO can be electrically tuned with the optically resonance of the metasurface $[96,97]$.

The evolution of nonlinear nanoresonators and metasurfaces based on dielectric and semiconductor materials is expected to grow in the next years since they hold the promise for small-footprint on-chip optical components for key functionalities such as active switching, optical isolation, routing, and frequency conversion. At the present state-of-the-art much remains to be explored on the fundamental side, such as new material systems and phenomena, new metasurface architectures, and further advancement of numerical methods to accurately model the nonlinear and tunable optical response of dielectric and semiconductor metasurfaces.

Author contribution: All the authors have accepted responsibility for the entire content of this submitted manuscript and approved submission.

Research funding: The authors acknowledge financial support by the Italian Ministry of University and Research through the PRIN Project "NOMEN" (proj. 2017MP7F8F), the European Community through the project "METAFAST" (H2020-FETOPEN-2018-2020 grant agreement no. 899673), the National Research Council Joint Laboratories program, Project No. SAC.AD002.026 (OMEN), the NATO through Science for Peace and Security 
(SPS) Programme, project “OPTIMIST” (SPS G5850) and the Army Research Laboratory under Cooperative Agreement Number W911NF-20-2-0078.

Conflict of interest statement: The authors declare no conflicts of interest regarding this article.

\section{References}

[1] A. I. Kuznetsov, A. E. Miroshnichenko, M. L. Brongersma, Y. S. Kivshar, and B. Luk'yanchuk, "Optically resonant dielectric nanostructures,” Science, vol. 354, no. 6314, p. aag2472, 2016.

[2] S. Kruk and Y. Kivshar, "Functional meta-optics and nanophotonics governed by Mie resonances," ACS Photonics, vol. 4, no. 11, pp. 2638-2649, 2017.

[3] D. Neshev and I. Aharonovich, "Optical metasurfaces: new generation building blocks for multi-functional optics," Light Sci. Appl., vol. 7, no. 1, p. 58, 2018.

[4] A. E. Minovich, A. E. Miroshnichenko, A. Y. Bykov, T. V. Murzina, D. N. Neshev, and Y. S. Kivshar, "Functional and nonlinear optical metasurfaces," Laser Photon. Rev., vol. 9 , no. 2, pp. 195-213, 2015.

[5] Y. Kivshar, "All-dielectric meta-optics and non-linear nanophotonics," Natl. Sci. Rev., vol. 5, pp. 144-158, 2018.

[6] K. Koshelev, A. Bogdanov, and Y. Kivshar, "Meta-optics and bound states in the continuum," Sci. Bull., vol. 64, no. 12, pp. 836-842, 2019.

[7] V. Zubyuk, L. Carletti, M. R. Shcherbakov, and S. S. Kruk, "Resonant dielectric metasurfaces in strong optical fields," Apl. Mater., vol. 9, no. 6, p. 060701, 2021.

[8] G. Li, S. Zhang, and T. Zentgraf, "Nonlinear photonic metasurfaces,” Nat. Rev. Mater., vol. 2, no. 5, p. 17010, 2017.

[9] L. Carletti, K. Koshelev, C. De Angelis, and Y. Kivshar, "Giant nonlinear response at the nanoscale driven by bound states in the continuum," Phys. Rev. Lett., vol. 121, no. 3, p. 033903 , 2018.

[10] K. Koshelev, Y. Tang, K. Li, D.-Y. Choi, G. Li, and Y. Kivshar, "Nonlinear metasurfaces governed by bound states in the continuum," ACS Photonics, vol. 6, no. 7, pp. 1639-1644, 2019.

[11] K. Koshelev, S. Kruk, E. Melik-Gaykazyan, et al., "Subwavelength dielectric resonators for nonlinear nanophotonics," Science, vol. 367, no. 6475, pp. 288-292, 2020.

[12] J. A. Schuller, E. S. Barnard, W. Cai, Y. C. Jun, J. S. White, and M. L. Brongersma, "Plasmonics for extreme light concentration and manipulation,” Nat. Mater., vol. 9, pp. 193-204, 2010.

[13] J. Z. Zhang and C. Noguez, "Plasmonic optical properties and applications of metal nanostructures," Plasmonics, vol. 3, pp. 127-150, 2008.

[14] V. F. Gili, L. Carletti, A. Locatelli, et al., "Monolithic AlGaAs second-harmonic nanoantennas," Opt. Express, vol. 24 no. 14, pp. 15965-15971, 2016.

[15] R. Camacho-Morales, M. Rahmani, S. Kruk, et al., "Nonlinear generation of vector beams from AlGaAs nanoantennas," Nano Lett., vol. 16, no. 11, pp. 7191-7197, 2016.

[16] S. Liu, M. B. Sinclair, S. Saravi, et al., "Resonantly enhanced second-harmonic generation using III-V semiconductor all-dielectric metasurfaces," Nano Lett., vol. 16, no. 9 , pp. 5426-5432, 2016.

[17] A. P. Anthur, H. Zhang, R. Paniagua-Dominguez, et al., "Continuous wave second harmonic generation enabled by quasi-bound-states in the continuum on gallium phosphide metasurfaces," Nano Lett., vol. 20, no. 12, pp. 8745-8751, 2020.

[18] L. Carletti, A. Zilli, F. Moia, et al., "Steering and encoding the polarization of the second harmonic in the visible with a monolithic $\mathrm{LiNbO}_{3}$ metasurface," ACS Photonics, vol. 8, no. 3 pp. 731-737, 2021.

[19] A. Fedotova, M. Younesi, J. Sautter, et al., "Second-harmonic generation in resonant nonlinear metasurfaces based on lithium niobate," Nano Lett., vol. 20, pp. 8608-8614, 2020.

[20] M. R. Shcherbakov, D. N. Neshev, B. Hopkins, et al., "Enhanced third-harmonic generation in silicon nanoparticles driven by magnetic response," Nano Lett., vol. 14, pp. 6488-6492, 2014.

[21] L. Xu, M. Rahmani, K. Z. Kamali, et al., "Boosting third-harmonic generation by a mirror-enhanced anapole resonator," Light Sci. Appl., vol. 7, no. 1, p. 44, 2018.

[22] Z. Liu, Y. Xu, Y. Lin, et al., "High-Q quasibound states in the continuum for nonlinear metasurfaces," Phys. Rev. Lett. vol. 123, no. 25, p. 253901, 2019.

[23] G. Grinblat, Y. Li, M. P. Nielsen, R. F. Oulton, and S. A. Maier, "Efficient third harmonic generation and nonlinear subwavelength imaging at a higher-order anapole mode in a single Germanium nanodisk," ACS Nano, vol. 11, no. 1 , pp. 953-960, 2017.

[24] S. V. Makarov, A. S. Zalogina, M. Tajik, et al., "Light-induced tuning and reconfiguration of nanophotonic structures," Laser Photon. Rev., vol. 11, no. 5, p. 1700108, 2017.

[25] C. Zou, J. Sautter, F. Setzpfandt, and I. Staude, "Resonant dielectric metasurfaces: active tuning and nonlinear effects," J. Phys. Appl. Phys., vol. 52, no. 37, 2019. https://doi.org/10 .1088/1361-6463/ab25ff

[26] A. M. Shaltout, V. M. Shalaev, and M. L. Brongersma, "Spatiotemporal light control with active metasurfaces," Science, vol. 364, no. 6442, p. eaat3100, 2019.

[27] Q. He, S. Sun, and L. Zhou, "Tunable/reconfigurable metasurfaces: physics and applications," Research, vol. 2019, pp. 1-16, 2019.

[28] C. W. Lee, H. J. Choi, and H. Jeong, "Tunable metasurfaces for visible and SWIR applications," Nano Convergence, vol. 7, no. 1, p. 3, 2020.

[29] D. Rocco, L. Carletti, R. Caputo, M. Finazzi, M. Celebrano, and C. De Angelis, "Switching the second harmonic generation by a dielectric metasurface via tunable liquid crystal," Opt. Express, vol. 28, no. 8, p. 12037, 2020.

[30] M. Celebrano, D. Rocco, M. Gandolfi, et al., "Optical tuning of dielectric nanoantennas for thermo-optically reconfigurable nonlinear metasurfaces," Opt. Lett., vol. 46, no. 10, p. 2453 , 2021.

[31] L. Carletti, D. de Ceglia, M. A. Vincenti, and C. De Angelis, "Self-tuning of second-harmonic generation in GaAs nanowires enabled by nonlinear absorption," Opt. Express, vol. 27, no. 22, p. 32480, 2019.

[32] X. Guo, Y. Ding, and X. Ni, "Electrically tunable second harmonic generation enhancement on a parametrically excited 
metasurface," in Conference on Lasers and Electro-Optics, OSA, 2020.

[33] A. Schirato, A. Mazzanti, R. P. Zaccaria, P. Nordlander, A. Alabastri, and G. D. Valle, "All-optically reconfigurable plasmonic metagrating for ultrafast diffraction management," Nano Lett., vol. 21, no. 3, pp. 1345-1351, 2021.

[34] M. Decker, C. Kremers, A. Minovich, et al., "Electro-optical switching by liquid-crystal controlled metasurfaces," Opt. Express, vol. 21, no. 7, pp. 8879-8885, 2013.

[35] H. Su, H. Wang, H. Zhao, T. Xue, and J. W. Zhang, "Liquid-crystal-based electrically tuned electromagnetically induced transparency metasurface switch," Sci. Rep., vol. 7, no. 1, pp. 1-7, 2017.

[36] F. Du, Y.-Q. Lu, and S.-T. Wu, "Electrically tunable liquid-crystal photonic crystal fiber,” Appl. Phys. Lett., vol. 85, no. 12, pp. 2181-2183, 2004.

[37] L. M. Blinov and V. G. Chigrinov, Electrooptic Effects in Liquid Crystal Materials, New York, Springer, 1994.

[38] J. Wu, Z. Shen, S. Ge, et al., "Liquid crystal programmable metasurface for terahertz beam steering," Appl. Phys. Lett., vol. 116, no. 13, p. 131104, 2020.

[39] I. Kim, W.-S. Kim, K. Kim, et al., "Holographic metasurface gas sensors for instantaneous visual alarms," Sci. Adv., vol. 7, no. 15, p. eabe9943, 2021.

[40] R. Caputo, L. De Sio, A. Veltri, C. Umeton, and A. V. Sukhov, "Development of a new kind of switchable holographic grating made of liquid-crystal films separated by slices of polymeric material," Opt. Lett., vol. 29, no. 11, pp. 1261-1263, 2004.

[41] E. P. Raynes, "Electro-optic and thermo-optic effects in liquid crystals," Phil. Trans. Roy. Soc. Lond. Math. Phys. Sci., vol. 309, no. 1507, pp. 167-178, 1983.

[42] A. Komar, Z. Fang, J. Bohn, et al., "Electrically tunable all-dielectric optical metasurfaces based on liquid crystals," Appl. Phys. Lett., vol. 110, no. 7, p. 071109, 2017.

[43] A. Komar, R. Paniagua-Domínguez, A. Miroshnichenko, et al., "Dynamic beam switching by liquid crystal tunable dielectric metasurfaces," ACS Photonics, vol. 5, no. 5, pp. 1742-1748, 2018.

[44] S.-Q. Li, X. Xu, R. M. Veetil, V. Valuckas, R. Paniagua-Domínguez, and A. I. Kuznetsov, "Phase-only transmissive spatial light modulator based on tunable dielectric metasurface," Science, vol. 364, no. 6445, pp. 1087-1090, 2019.

[45] S. T. Wu and C. S. Wu, "High-speed liquid-crystal modulators using transient nematic effect," J. Appl. Phys., vol. 65, no. 2, pp. 527-532, 1989.

[46] M. Gandolfi, G. Benetti, C. Glorieux, C. Giannetti, and F. Banfi, "Accessing temperature waves: a dispersion relation perspective," Int. J. Heat Mass Tran., vol. 143, p. 118553, 2019.

[47] M. Gandolfi, C. Giannetti, and F. Banfi, "Temperonic crystal: a superlattice for temperature waves in graphene," Phys. Rev. Lett., vol. 125, no. 26, p. 265901, 2020.

[48] G. P. Zograf, M. I. Petrov, S. Makarov, and Y. Kivshar “All-dielectric thermonanophotonics," pp.1-92, 2021. arXiv:2104.01964.

[49] T. Stoll, P. Maioli, A. Crut, et al., "Time-resolved investigations of the cooling dynamics of metal nanoparticles: impact of environment," J. Phys. Chem. C, vol. 119, no. 22, pp. 12757-12764, 2015.
[50] S. Danesi, M. Gandolfi, L. Carletti, et al., "Photo-induced heat generation in non-plasmonic nanoantennas," Phys. Chem. Chem. Phys., vol. 20, no. 22, pp. 15307-15315, 2018.

[51] M. Gandolfi, F. Banfi, and C. Glorieux, "Optical wavelength dependence of photoacoustic signal of gold nanofluid," Photoacoustics, vol. 20, p. 100199, 2020.

[52] G. P. Zograf, M. I. Petrov, D. A. Zuev, et al., "Resonant nonplasmonic nanoparticles for efficient temperature-feedback optical heating," Nano Lett., vol. 17, no. 5, pp. 2945-2952, 2017.

[53] S. Makarov, S. Kudryashov, I. Mukhin, et al., "Tuning of magnetic optical response in a dielectric nanoparticle by ultrafast photoexcitation of dense electron-hole plasma," Nano Lett., vol. 15, no. 9, pp. 6187-6192, 2015.

[54] M. Aouassa, E. Mitsai, S. Syubaev, et al., "Temperature-feedback direct laser reshaping of silicon nanostructures,” Appl. Phys. Lett., vol. 111, no. 24, p. 243103 , 2017.

[55] M. A. Kats, R. Blanchard, P. Genevet, et al., "Thermal tuning of mid-infrared plasmonic antenna arrays using a phase change material," Opt. Lett., vol. 38, no. 3, pp. 368-370, 2013.

[56] M. Rahmani, L. Xu, A. E. Miroshnichenko, et al., "Reversible thermal tuning of all-dielectric metasurfaces," Adv. Funct. Mater., vol. 27, no. 31, p. 1700580, 2017.

[57] K. Z. Kamali, L. Xu, J. Ward, et al., "Reversible image contrast manipulation with thermally tunable dielectric metasurfaces," Small, vol. 15, no. 15, p. 1805142, 2019.

[58] D. G. Baranov, S. V. Makarov, A. E. Krasnok, P. A. Belov, and A. Alù, "Tuning of near- and far-field properties of all-dielectric dimer nanoantennas via ultrafast electron-hole plasma photoexcitation," Laser Photon. Rev., vol. 10, no. 6, pp. 1009-1015, 2016.

[59] M. Gandolfi, A. Crut, F. Medeghini, et al., "Ultrafast thermo-optical dynamics of plasmonic nanoparticles," J. Phys. Chem. C, vol. 122, no. 15, pp. 8655-8666, 2018.

[60] T. Skauli, P. S. Kuo, K. L. Vodopyanov, et al., "Improved dispersion relations for $\mathrm{GaAs}$ and applications to nonlinear optics," J. Appl. Phys., vol. 94, no. 10, pp. 6447-6455, 2003.

[61] M. C. Downer and C. V. Shank, "Ultrafast heating of silicon on sapphire by femtosecond optical pulses," Phys. Rev. Lett., vol. 56, no. 7, pp. 761-764, 1986.

[62] S. Abdollahramezani, O. Hemmatyar, H. Taghinejad, et al., "Tunable nanophotonics enabled by chalcogenide phase-change materials," Nanophotonics, vol. 9, no. 5, pp. 1189-1241, 2020.

[63] B. J. Eggleton, B. Luther-Davies, and K. Richardson, “Chalcogenide photonics," Nat. Photonics, vol. 5, no. 3, pp. 141-148, 2011.

[64] K. Guo, K. Zhou, and Z. Guo, "Tunable second harmonic generation from bianisotropic plasmonic metamolecule via utilizing phase change materials," J. Appl. Phys., vol. 128, no. 13, p. 133104, 2020.

[65] H. L. Liu, Z. Zhibo, S. Zhenchen, G. Tian, and W. Xijun, "Dynamically manipulating third-harmonic generation of phase change material with gap-plasmon resonators," Opt. Lett., vol. 44, no. 20, p. 5053, 2019.

[66] J. Liu, S. Liu, and J. Wei, "Origin of the giant optical nonlinearity of $\mathrm{Sb}_{2} \mathrm{Te}_{3}$ phase change materials," Appl. Phys. Lett., vol. 97, no. 26, p. 261903, 2010. 
[67] R. W. Boyd, Nonlinear Optics, New York, Academic Press, 2008.

[68] G. I. Petrov, V. V. Yakovlev, and J. A. Squier, "Nonlinear optical microscopy analysis of ultrafast phase transformation in vanadium dioxide," Opt. Lett., vol. 27, no. 8, p. 655, 2002.

[69] S. Lysenko, A. Rua, F. Fernandez, and H. Liu, "Optical nonlinearity and structural dynamics of $\mathrm{VO}_{2}$ films," J. Appl. Phys., vol. 105, no. 4, p. 043502, 2009.

[70] S. Lysenko, V. Vikhnin, A. Rua, F. Fernandez, and H. Liu, "Critical behavior and size effects in light-induced transition of nanostructured $\mathrm{VO}_{2}$ films," Phys. Rev. $B$, vol. 82 , no. 20 , p. 205425, 2010.

[71] A. Tognazzi, A. Locatelli, M. A. Vincenti, C. Giannetti, and C. De Angelis, "Tunable optical antennas using vanadium dioxide metal-insulator phase transitions," Plasmonics, vol. 14, no. 5, pp. 1283-1288, 2019.

[72] T. Cao, K. Liu, Y. Tang, J. Deng, K. Li, and G. Li, "A high-index $\mathrm{Ge}_{2} \mathrm{Sb}_{2} \mathrm{Te}_{5}$-based fabry-perot cavity and its application for third-harmonic generation," Laser Photon. Rev., vol. 13, no. 7, p. 1900063, 2019.

[73] M. Marni and D. de Ceglia, "Strong modulation of absorption and third-harmonic generation in resonant metasurfaces based on $\mathrm{VO}_{2}$," in Proceedings of the 9th International Conference on Photonics, Optics and Laser Technology, SCITEPRESS - Science and Technology Publications, 2021.

[74] N. A. Charipar, H. Kim, S. A. Mathews, and A. Piqué, "Broadband terahertz generation using the semiconductor-metal transition in $\mathrm{VO}_{2}$, , AIP Adv., vol. 6, no. 1, p. 015113, 2016.

[75] M. Esaulkov, P. Solyankin, A. Sidorov, et al., "Emission of terahertz pulses from vanadium dioxide films undergoing metal-insulator phase transition," Optica, vol. 2, no. 9, p. 790, 2015.

[76] M. Rudé, V. Mkhitaryan, A. E. Cetin, et al., "Ultrafast and broadband tuning of resonant optical nanostructures using phase-change materials," Adv. Opt. Mater., vol. 4, no. 7, pp. 1060-1066, 2016.

[77] M. Wuttig, H. Bhaskaran, and T. Taubner, "Phase-change materials for non-volatile photonic applications," Nat. Photonics, vol. 11, no. 8, pp. 465-476, 2017.

[78] Y. Wang, P. Landreman, D. Schoen, et al., "Electrical tuning of phase-change antennas and metasurfaces," Nat. Nanotechnol., vol. 16, pp. 667-672, 2021.

[79] M. Lawrence, D. R. Barton, and J. A. Dionne, "Nonreciprocal flat optics with silicon metasurfaces," Nano Lett., vol. 18, no. 2, pp. 1104-1109, 2018.

[80] X. Guo, Y. Ding, Y. Duan, and X. Ni, "Nonreciprocal metasurface with space-time phase modulation," Light Sci. Appl., vol. 8, p. 123, 2019.

[81] B. Slovick, L. Zipp, and S. Krishnamurthy, "Indium phosphide metasurface with enhanced nonlinear absorption," Sci. Rep., vol. 7, no. 1, p. 17245, 2017.

[82] G. D. Valle, B. Hopkins, L. Ganzer, et al., "Nonlinear anisotropic dielectric metasurfaces for ultrafast nanophotonics," $A C S$ Photonics, vol. 4, no. 9, pp. 2129-2136, 2017.
[83] T. Ning, X. Li, Z. Zhang, et al., “Ultimate conversion efficiency of second harmonic generation in all-dielectric resonators of quasi-BICs in consideration of nonlinear refraction of dielectrics," Opt. Express, vol. 29, no. 11, p. 17286, 2021.

[84] B. R. Bennett, R. A. Soref, and J. A. Del Alamo, "Carrier-induced change in refractive index of InP, GaAs and InGaAsP," IEEE J. Quant. Electron., vol. 26, no. 1, pp.113-122, 1990.

[85] M. R. Shcherbakov, P. P. Vabishchevich, A. S. Shorokhov, et al., "Ultrafast all-optical switching with magnetic resonances in nonlinear dielectric nanostructures," Nano Lett., vol. 15, no. 10, pp. 6985-6990, 2015.

[86] P. Franceschini, L. Carletti, A. P. Pushkarev, F. Preda, A. Perri, A. Tognazzi, et al., "Tuning the ultrafast response of fano resonances in halide perovskite nanoparticles," ACS Nano, vol. 14, pp. 13602-13610, 2020.

[87] M. R. Shcherbakov, K. Werner, Z. Fan, N. Talisa, E. Chowdhury, and G. Shvets, "Photon acceleration and tunable broadband harmonics generation in nonlinear time-dependent metasurfaces," Nat. Commun., vol. 10, no. 1, p. 1345, 2019.

[88] Z. Hayran and F. Monticone, "Capturing broadband light in a compact bound state in the continuum," ACS Photonics, vol. 8 , no. 3, pp. 813-823, 2021.

[89] P. Gutruf, C. Zou, W. Withayachumnanku, M. Bhaskaran, S. Sriram, and C. Fumeaux, "Mechanically tunable dielectric resonator metasurfaces at visible frequencies," ACS Nano, vol. 10, no. 1, pp. 133-141, 2016.

[90] S. M. Kamali, E. Arbabi, A. Arbabi, Y. Horie, and A. Faraon, "Highly tunable elastic dielectric metasurface lenses," Laser Photon. Rev., vol. 10, no. 6, pp. 1002-1008, 2016.

[91] A. She, S. Zhang, S. Shian, D. R. Clarke, and F. Capasso, "Adaptive metalenses with simultaneous electrical control of focal length, astigmatism, and shift," Sci. Adv., vol. 4, no. 2, pp. 1-8, 2018.

[92] A. Karvounis, J.-Y. Ou, W. Wu, K. F. McDonald, and N. I. Zheludev, "Nano-optomechanical nonlinear dielectric metamaterials,” Appl. Phys. Lett., vol. 107, no. 19, p. 191110, 2015.

[93] I. A. Ajia, J.-Y. Ou, N. J. Dinsdale, et al., “Gigahertz nano-optomechanical resonances in a dielectric SiC-membrane metasurface array," Nano Lett., vol. 21, pp. 4563-4569, 2021.

[94] G. Soavi, G. Wang, H. Rostami, et al., "Broadband, electrically tunable third-harmonic generation in graphene," Nat. Nanotechnol., vol. 13, no. 7, pp. 583-588, 2018.

[95] Y. Cheng, H. Hong, H. Zhao, et al., "Ultrafast optical modulation of harmonic generation in two-dimensional materials," Nano Lett., vol. 20, pp. 8053-8058, 2020.

[96] A. Howes, W. Wang, I. Kravchenko, and J. Valentine, “Dynamic transmission control based on all-dielectric Huygens metasurfaces," Optica, vol. 5, no. 7, p. 787, 2018.

[97] A. Forouzmand, M. M. Salary, S. Inampudi, and H. Mosallaei, "A tunable multigate indium-tin-oxide-assisted all-dielectric metasurface," Adv. Opt. Mater., vol. 6, no. 7, p. 1701275 , 2018. 